\section{Check for updates}

Cite this: J. Anal. At. Spectrom., 2019, 34, 1900

Received 28th May 2019

Accepted 25th July 2019

DOI: 10.1039/c9ja00186g

rsc.li/jaas

\title{
Performance of sp-ICP-TOFMS with signal distributions fitted to a compound Poisson model
}

\author{
Lyndsey Hendriks, Alexander Gundlach-Graham (D) and Detlef Günther
}

\begin{abstract}
Accurate separation of signals from individual nanoparticles (NPs) from background ion signals is decisive to correct sizing and number-concentration determinations in single-particle (sp) ICP-MS analyses. In typical sp-ICP-MS approaches, NP signals are identified via outlier analysis based on the assumption of normally distributed (i.e. Gaussian) or Poisson-distributed background signals. However, for sp-ICP-MS with a Time-of-Flight (TOF) mass spectrometer that digitizes MS signal by fast analog-to-digital conversion (ADC), the background ion signals are neither Gaussian nor Poisson. Instead, steady-state ion signals with ICP-TOFMS follow a compound Poisson distribution that reflects noise contributions from Poissondistributed arrival of ions and gain statistics of microchannel-plate-based ion detection. Here, we characterize this compound Poisson distribution with Monte Carlo simulations to establish net critical values $\left(L_{C(A D C)}\right)$ as detection decision levels for the discrimination of discrete NPs in sp-ICP-TOFMS analyses. We apply $L_{C(A D C)}$ to the analysis of gold-silver core-shell nanoparticles (Au-Ag NPs), and compare these results to conventional sigma-based NP-detection thresholds. Additionally, we investigate how accurate modelling of the compound Poisson TOFMS signal distribution enables separation of overlapping background and NP distributions; we demonstrate accurate size measurement of $20 \mathrm{~nm}$ Au NPs that have mean signal intensity of less than four counts.
\end{abstract}

\section{Introduction}

\subsection{Sp-ICP-MS and its challenges regarding the detection of} increasingly smaller NPs

Coincident with a strong drive in nanotechnology to explore the promises of smaller and smaller nanoparticles (NPs)—such as $3 \mathrm{~nm}$ supermagnetic iron oxide NPs as positive MRI contrast agents $^{1}$ or gold NPs for targeted cancer therapy $y^{2,3}$-continued efforts in single-particle inductively coupled plasma mass spectrometry (sp-ICP-MS) are aimed at detecting the smallest particle size. Indeed, the small size and high surface-to-volume ratio of nanoparticles compared to their ionic counterparts, strongly influence their physiochemical and toxicokinetic properties., ${ }^{\mathbf{4 5}}$

In sp-ICP-MS, accurate measurement of NP size or particle number concentration (PNC) is only possible if NP signals are distinguishable from background signals,${ }^{6-8}$ which means that NP-size detection limits can only be improved through increasing the NP-to-dissolved background signal ratio. To accomplish this, either the instrument sensitivity can be increased or the dissolved background can be reduced. For example, dissolved background can be reduced through simple sample dilution, ion-exchange purification, ${ }^{\mathbf{9}, 10}$ NP extraction, ${ }^{11}$ or separation methods. ${ }^{\mathbf{1 2}-14}$ Despite the effectiveness of the

Department of Chemistry and Applied Biosciences, ETH Zurich, Zurich, Switzerland. E-mail: graham@inorg.chem.ethz.ch different approaches to remove dissolved analyte species, there will always be a non-zero background and, for the detection of small NPs that produce low ion-count signals, analyte sensitivity becomes the major limitation. Recall that the mass content scales with the cube of the diameter, so a $10 \mathrm{~nm}$ diameter $\mathrm{Au}$ $\mathrm{NP}$, which is only 5 times smaller in diameter than a $50 \mathrm{~nm}$ diameter $\mathrm{Au} \mathrm{NP}$, contains little mass ( $\sim 10 \mathrm{ag})$ compared to a $50 \mathrm{~nm}$ Au NP ( 1260 ag). To date, the smallest detectable Au NPs are between $4-7 \mathrm{~nm}$ in diameter, ${ }^{15,16}$ and in order to measure NPs with half this diameter, one would require 8-times higher sensitivity. Near the size detection limit, particle signals are, by definition, similar in signal intensity to background signals, so data processing algorithms used to discriminate NP and dissolved background signals are critical. Currently, sp-ICPMS NP identification algorithms are either based on outlier detection ${ }^{17-20}$ or deconvolution ${ }^{21}$ of the dissolved and particulate fractions.

\subsection{Common approaches for finding NPs in sp-ICP-MS}

The most common strategy applied in sp-ICP-MS to find NPs is to treat NP signals as outliers, and the dissolved background signals as normal objects. In this approach, a particle event is detected as an outlier if its signal intensity exceeds a defined threshold. Usually, the NP-detection threshold is determined with an iterative algorithm, which detects NPs from the dissolved background when they exceed $\mu+n \sigma$, where $\mu$ is the mean of the whole dataset and $n \sigma$ an integer multiple times the 
standard deviation. ${ }^{7,8,19,20}$ Alternatively, $k$-means clustering has also been discussed as an approach to separate the data into dissolved and NP fractions, ${ }^{22}$ but has not been widely used. More recently, a data evaluation approach based on Poisson statistics has been applied to determine thresholds to identify the start and end of transient signals from single NPs for data acquired with microsecond time resolution. ${ }^{23}$

The basic steps of iterative outlier analysis for sp-ICP-MS are: (1) the entire dataset is averaged, (2) all events that are above $\mu+$ $n \sigma$ are recognized as outliers and removed from the dataset, (3) $\mu$ and $\sigma$ of the remaining dataset are recalculated and new outliers are collected, (4) iterations continue until no data are detected above the final $\mu+n \sigma$ threshold. Importantly, the NPdetection limit as defined here is, in fact, a decision level based solely on controlling the number of false positives (i.e. dissolved signals identified as NPs): at the NP-detection limit, by definition (assuming homoscedasticity), 50\% of analyte NPs would be recorded as false negatives. ${ }^{24}$ Because we have no a priori information on shape, size, or predicted signal variance from analyte NP populations, identification of NP signals is best achieved with a decision level. In the case of $\mu+n \sigma$ detection decisions, in which background signal is assumed to be normally distributed (i.e. Gaussian), the $\mu+n \sigma$ method should find outliers (i.e. NPs) with false positive rates of $0.135 \%, 0.0032 \%$, or $0.000028 \%$ (280 ppb) for $n$ values of 3, 4, or 5 , respectively. At low background ion-count rates, $n \sigma$ thresholds do not accurately predict false positive rates because counting statistics dominate and the background more closely matches a Poisson distribution for pulse counting MS detectors. ${ }^{19}$

Throughout the sp-ICP-MS literature, there is no consensus on what integer value of sigma should be used to discriminate NP signals from dissolved signals. Studies independently report that $3 \sigma,^{25} 5 \sigma,^{15,20}$ or even $7 \sigma^{26}$ threshold criteria provide best NPdetection accuracy. The range of NP-detection criteria routinely used is evidence of the importance of accurate background noise characterisation. False-positive rates for NP detection thresholds above $5 \sigma$ do not provide (statistically) more discrimination power for the PNCs typically measured; instead, these more restrictive NP-detection thresholds are used because the background signals cannot be fully described by a normal distribution.

\subsection{Critical values as NP-detection decisions}

As will be further discussed, sp-ICP-TOFMS signals measured with a fast analog-to-digital conversion (ADC) detection system are neither Gaussian nor Poisson distributed, but are best characterized as a compound Poisson distribution. This compound Poisson distribution incorporates signal variance from Poissondistributed arrival of ions and gain statistics of microchannelplate (MCP) based ion detection. ${ }^{27}$ Therefore, any NP-detection threshold used for sp-ICP-TOFMS should take into account the shape of the compound Poisson distribution to establish detection thresholds that have a reliable false-positive rates.

In our treatment of detection criteria for sp-ICP-TOFMS, we follow the definition of detection developed by Currie ${ }^{28,29}$ and adopted by IUPAC, ${ }^{30}$ in which the minimum detectable signal (i.e. the detection decision) is termed the critical value $L_{\mathrm{c}}$. In spICP-MS, the critical value is equivalent to the NP-detection threshold: it is the signal value above which one claims all signals come from NPs. ${ }^{19}$ According to Currie, detection is, in principle, a judgement on whether an observed signal originates from the analysed sample or whether it is caused by blank or background signal. If one understands or can predict the background distribution, then one can set a critical value above which there is only a small probability that a signal comes from the background, i.e. it is a false positive. Currie defines the false positive rate as the term $\alpha$. For conventional steady-state signals, an $\alpha$ of 0.05 (5\%) is custom. ${ }^{29}$ However, in sp-ICP-MS, background events typically greatly outnumber NP events, so more conservative $\alpha$ values of $0.1 \%$ or even $0.01 \%$ are suggested. ${ }^{19,27}$ For example, in sp-ICP-MS measurements, often less than $10 \%$ of the dataset is occupied by NP signals. Assuming a background : NP event ratio of 10000 : 1000 and NP-detection with $\alpha$ values of $5 \%$ and $0.1 \%$, one would expect 500 and 10 signals falsely recognized as NP signals, respectively, which would lead to errors of $+50 \%$ or $+1 \%$ in particle number. Consequently, the assignment of robust NP-detection critical values in sp-ICP-MS depends both on the ability to accurately predict and describe background distributions, as well as on the selection of an appropriate $\alpha$ value for the expected ratio of recorded NP events to background.

In the following discussion, we demonstrate how to accurately characterize compound Poisson background distributions in sp-ICP-TOFMS and evaluate the robustness of compound-Poisson-derived net critical values $\left(L_{\mathrm{C}(\mathrm{ADC})}\right)$, for use as NP-detection thresholds. We specifically explore the use $L_{\mathrm{C}(\mathrm{ADC})}$ for the challenging case of detecting of small Au NPs (20 and $30 \mathrm{~nm}$ in diameter) that produce low-ion signals of similar magnitude to signals from the dissolved background.

\section{Experimental}

\subsection{Materials}

Dilute suspensions of commercially available monodisperse $20 \mathrm{~nm} \mathrm{Au} \mathrm{NPs}\left(18.9 \pm 1.5 \mathrm{~nm}, 0.052 \mathrm{mg} \mathrm{mL}{ }^{-1}\right.$ stabilized in $2 \mathrm{mM}$ citrate) and $\mathrm{Au}-\mathrm{Ag}$ core-shell NPs (total diameter $61 \pm$ $1 \mathrm{~nm}, \mathrm{Ag}$ shell thickness $15 \mathrm{~nm}$, Au core diameter $30 \pm 3 \mathrm{~nm}$, $0.021 \mathrm{mg} \mathrm{mL}^{-1}$ stabilized in $2 \mathrm{mM}$ citrate) from NanoComposix (San Diego, CA, USA) were analyzed in this study (see Table 1 for specifications). The stock suspensions were stored in the refrigerator at $4-5{ }^{\circ} \mathrm{C}$ and allowed to warm to room temperature for 15 minutes, and then sonicated for 2 minutes prior to dilution. All dilutions were carried out in ultra-high purity (UHP) water.

Microdroplets were used for NP mass calibration and calibration solutions were prepared by diluting commercially available single-element standard solutions of $\mathrm{Au}$ and $\mathrm{Ag}$ (Inorganic Ventures, USA) in $1 \%$ sub-boiled $\mathrm{HNO}_{3}$ and $1 \% \mathrm{HCl}$ (VWR Chemicals, USA). $100 \mu \mathrm{g} \mathrm{L}^{-1}$ of Cs was also added to microdroplet solutions; Cs signals were used as a tracer to identify microdroplet signals. All dilutions were performed gravimetrically using a balance (Mettler AE240, Mettler-Toledo, Greifensee, Switzerland). 
Table 1 Au-Ag coreshell NPs: manufacturers specifications and calculated variables

Total diameter (TEM)

Au core diameter (TEM)

Particle concentration

Mass concentration $(\mathrm{Au})$

Mass concentration (Ag)

Shell thickness (calculated from total diameter)

Mass Au core (calculated from Au-core diameter)

Mass Ag shell (calculated from volume)

Mass Ag shell (calculated from mass concentration)
$61 \pm 6 \mathrm{~nm}$

$30 \pm 3 \mathrm{~nm}$

$1.5 \times 10^{10}$ particles per $\mathrm{mL}$

$5 \mu \mathrm{g} \mathrm{mL} \mathrm{L}^{-1}$

$0.016 \mu \mathrm{g} \mathrm{mL}^{-1}$

$15.5 \pm 4 \mathrm{~nm}$

$0.28 \pm 0.08 \mathrm{fg}$

$1.13 \pm 0.4 \mathrm{fg}$

$1.07 \mathrm{fg}$

\subsection{S(T)EM measurements}

The $20 \mathrm{~nm} \mathrm{Au} \mathrm{NPs} \mathrm{were} \mathrm{characterized} \mathrm{by} \mathrm{scanning} \mathrm{(trans-}$ mission) electron microscopy (S(T)EM) to assess their size distributions. Samples were prepared by pipetting a few microliters of the stock NP solution onto a glow-discharged carbon foil on copper grids. After letting the particles adsorb on the foil for $30 \mathrm{~s}$, the excess NP solution was blotted off using filter paper and the samples were left to air dry. Images were recorded using an ultra-high resolution SEM (FEI Magellan 400 FEG, ThermoFisher Scientific, Eindhoven, Netherlands), operated at $30 \mathrm{kV}$ in immersion lens mode. Images were simultaneously recorded with a secondary electron detector (SE) as well as by the S(T)EM detector in bright field (BF) and high angle annual dark field (HAAF) mode. Image processing was performed using the BF signal, but $\mathrm{SE}$ images are shown for better illustration in Fig. 6. Images were analyzed for individual particle sizes in Matlab (ver.R2017b, Mathworks Inc., MA, USA) using image processing procedures including pattern recognition and distance calibration (pixel per $\mu \mathrm{m}$ ) based on the pixel size information provided in the metadata.

\subsection{Dual sample introduction experimental design}

In this study, we used the dual sample introduction setup developed by Ramkorum-Schmidt et al. ${ }^{31}$ in combination with an ICP-TOFMS instrument (icpTOF 2R, TOFWERK AG, Switzerland), as already described elsewhere. ${ }^{32,33}$ Operating parameters for both the dual sample introduction setup and the ICPTOFMS are provided in Table 2. Importantly, because the TOF mass analyzer provides simultaneous full mass spectrum detection at a high speed (25.136 $\mathrm{kSpectra}$ per s), multi-element NPs can be quantitatively measured. ${ }^{34-38}$ Online microdroplet calibration was used for NP mass quantification. ${ }^{32,33}$ In brief, NP-containing solutions were introduced through the nebulizer, while microdroplets composed of the calibration solution were produced by a microdroplet generator $(50 \mu \mathrm{m}$ diameter autodrop pipette, AD-KH-501-L6; MD-E-3000 dispensing system, Microdrop Technologies GmbH, Germany) and introduced concurrently into the ICP via a falling tube filled with a He/Ar gas mixture to accelerate droplet desolvation. ${ }^{39}$ Sensitivities recorded from microdroplets introduced concurrently with the analyte $\mathrm{Au}-\mathrm{Ag}$ or Au NPs were used to calibrate mass of the NPs. In this way, online microdroplet calibration provides direct compensation for any long-term sensitivity drift. The ICPTOFMS instrument was tuned for highest sensitivity for Ag and
Au. The collision/reaction cell was pressurized with $1 \mathrm{~mL} \mathrm{~min}{ }^{-1}$ He to promote collisional focussing and improve sensitivity.

\subsection{Data evaluation}

Averaged TOF mass spectra were recorded at a time resolution of $1.738 \mathrm{~ms}$ and stored in the HDF5 file format. After data acquisition, TOFMS data were processed in TOFWARE (ver. 2.5.11, TOFWERK, run in Igor-Pro 7 environment) to verify mass calibration and to export time traces of integrated mass spectral intensities of analyte isotopes and the microdroplet tracer (i.e. ${ }^{107} \mathrm{Ag}^{+},{ }^{109} \mathrm{Ag}^{+},{ }^{133} \mathrm{Cs}^{+}$and ${ }^{197} \mathrm{Au}^{+}$) as .csv files. Further data processing was carried out in LabVIEW (ver. 17.0.1f3, National Instruments, TX, USA).

Monte Carlo simulations were used to fit ICP-TOFMS background signal distributions to a compound Poisson model in

Table 2 Operating conditions for the dual sample introduction setup and ICP-TOFMS

\section{Microdroplet Introduction}

Droplet diameter

Droplet frequency

Ag concentration in droplets

Au concentration in droplets

He gas flow in falling tube

Ar gas flow in falling tube

$42.8 \mu \mathrm{m}$

$40 \mathrm{~Hz}$

$105 \mu \mathrm{g} \mathrm{L}^{-1}$

$96 \mu \mathrm{g} \mathrm{L}^{-1}$

$0.56 \mathrm{~L} \mathrm{~min}^{-1}$

$0.15 \mathrm{~L} \mathrm{~min}^{-1}$

Pneumatic nebulizer

Nebulizer gas (Ar)

Solution uptake rate

$0.82 \mathrm{~L} \mathrm{~min}^{-1}$

$\sim 600 \mu \mathrm{L} \mathrm{m^{-1 }}$

\section{ICP conditions}

Intermediate gas flow (Ar)

Outer gas flow (Ar)

Power

Sampling position

Collision/reaction cell (He)

$$
\begin{aligned}
& 0.8 \mathrm{~L} \mathrm{~min}^{-1} \\
& 15 \mathrm{~L} \mathrm{~min}^{-1} \\
& 1550 \mathrm{~W} \\
& 5 \mathrm{~mm} \text { above load coil } \\
& 1 \mathrm{~mL} \mathrm{~min}
\end{aligned}
$$

\section{TOFMS conditions}

TOF spectral acquisition rate ${ }^{a}$ Averaged spectrum acquisition rate $^{b}$

$25.316 \mathrm{kHz}$

$575.36 \mathrm{~Hz}(1.738 \mathrm{~ms})$

${ }^{a}$ In order to increase the sensitivity for $\mathrm{Au}$ and $\mathrm{Ag}$, the TOF spectral acquisition rate was adjusted from its standard value of $21.739 \mathrm{kHz}$ to $25.316 \mathrm{kHz}$, which increased sensitivity of $30 \%$, but introduced a high- $m / z$ cut-off above 200 Th. ${ }^{b}$ Averaged mass spectra are composed of data summed from 44 full mass spectra collected every $39.5 \mu \mathrm{s}$. Note that, for continuous liquid sample introduction of $\mathrm{Au}$ (see Fig. 1), the averaged spectrum acquisition rate was of $2.024 \mathrm{~ms}$ (i.e. 44 mass spectra at $21.739 \mathrm{kHz}$ ). 
the same manner as previously described.$^{27}$ Briefly, ICP-TOFMS data was truncated above the $90 \%$-quantile (or at 3 counts, whichever is larger) in order to minimize the number of NP signals in the dataset. The background data was then iteratively modelled as a compound Poisson distribution in which the TOFMS signal acquisition is considered to be the sum of a Poisson-distributed number of ions that each sample the measured single-ion-signal (SIS) distribution of the ADC-based TOF detection system. Fitting of the background TOFMS data distribution was done with a sum of squared error (SSE) minimization procedure that globally samples a range of background count rates $\left(\lambda_{\text {bkgd }}\right)$ and number of acquisitions $\left(N_{\text {acq }}\right)$ to estimate values that best describe the measured data. Critical values $\left(L_{\mathrm{C}(\mathrm{ADC})}\right)$ for NP detection were also calculated by Monte Carlo simulation with an in-house written LabVIEW program (available upon request) to approximate the relationship between $L_{\mathrm{C}(\mathrm{ADC})}$ and $\lambda_{\text {bkgd }}$ relationship for a given false-positive rate $(\alpha){ }^{27}$ Because $L_{\mathrm{C}(\mathrm{ADC})}$ depends on the shape of the SIS distribution of the TOFMS detection system, we measured the SIS histogram prior to sp-ICP-TOFMS analysis. To assign a value to $L_{\mathrm{C}(\mathrm{ADC})}$ for a given analysis, we used $\lambda_{\mathrm{bkgd}}$ determined by Monte Carlo fitting of the data and the derived expression for $L_{\mathrm{C}(\mathrm{ADC})}$. Subtraction of Monte-Carlo simulated background from measured signal distributions was performed in LabVIEW and results were saved as frequency histograms. Processed sp-ICPTOFMS data were plotted with OriginPro (ver 8.6.0, OriginLab Corp., MA, USA) and final figures were assembled in Adobe Illustrator (ver. 16.2.0, Adobe Systems Inc., USA).

For comparison of conventional NP-detection threshold determination methods (i.e. $\mu+3 \sigma$ and $\mu+5 \sigma$ ) with our compound-Poisson-based critical value approach, iterative outlier analysis was performed in Matlab. Due to an abundance of zero-count signals in the TOFMS data, signals with zero counts were removed from the dataset prior to outlier analysis. Alternative to outlier analysis, we found that fitting signal histograms with a Gaussian function provided a better estimation of $\mu$ and $\sigma$. Gaussian fitting of the background was also performed in Matlab.

NP mass calibration was carried out using analyte sensitivities determined from the microdroplets. The signals from at least 500 microdroplets for each sp-ICP-TOFMS measurement were recorded and averaged to determine the average singledroplet signal. Microdroplets for each measurement were sizecalibrated based on imaging dispensed droplets with a camera with known magnification and CMOS sensor pixel size. ${ }^{40}$ Then, as the microdroplet size and content are known, the average sensitivity in counts per unit mass of analyte in each droplet can readily be determined. ${ }^{41} \mathrm{NP}$ diameter was calculated assuming spherical geometry of the NPs and bulk density of the NP metal.

\section{Results and discussion}

\subsection{Compound Poisson modelling of TOFMS signals}

In this work, we investigate how the description of ICP-TOFMS signals as being compound Poisson distributed affects the performance of sp-ICP-TOFMS for the detection of NPs with lowcount signals. To illustrate the significance of this compound Poisson distribution, in Fig. 1 we present frequency histograms for ICP-TOFMS signals from continuous aspiration of $0.1 \mathrm{ng} \mathrm{g}^{-1}$ and $1 \mathrm{ng} \mathrm{g}^{-1} \mathrm{Au}$ solutions collected at a time resolution of 2.024 ms. For both of these histograms, we determined the count rate $\left(\lambda_{\text {bkgd }}\right)$ of the compound Poisson distribution that best describes the measured signal distributions. As seen in Fig. 1, the modelled distributions match the measured signal distributions extremely well, which is an indicator that our compound Poisson model accounts for the major sources of noise in ICP-TOFMS measurements. Furthermore, Fig. 1a shows the usual shape of low-count-rate ICP-TOFMS signal distributions, which have a high frequency zero-count bin and a right-skewed shape. Clearly, this signal distribution would not be adequately described as Gaussian or Poisson. On the other hand, in Fig. 1b
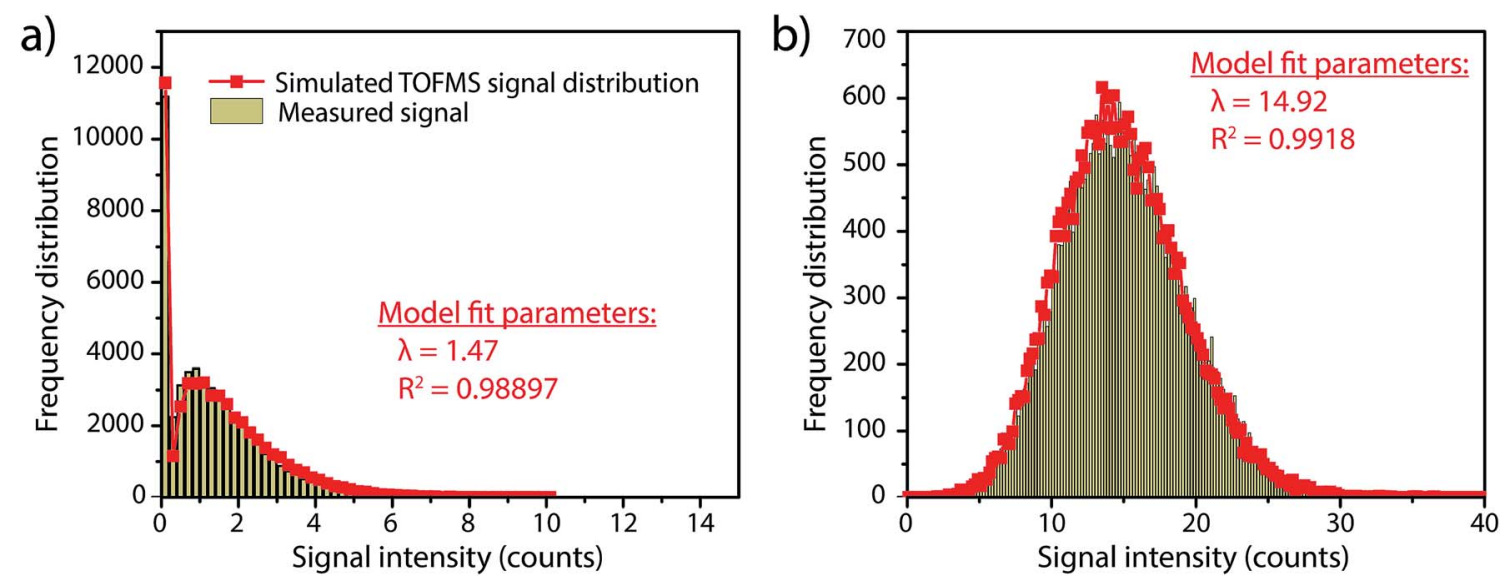

Fig. 1 (a) Histogram of low count signals recorded from a $0.1 \mathrm{ng} \mathrm{g}^{-1}$ Au solution presents an unusual signal distribution shape with a large fraction of zeros and a right skewed shape; this shape can be modelled as a compound Poisson distribution that takes into account Poissondistributed ion arrival and signal variation due to gain statistics of the TOF detection system. (b) Histogram of high-count signals recorded from a $1 \mathrm{ng} \mathrm{g}^{-1}$ Au solution shows an approximate Gaussian distribution because counting statistics noise dominates. In both cases, a Monte Carlo simulation that models the compound Poisson distribution of TOFMS data demonstrates an excellent match to the measured distributions. 
it is apparent that, while a compound Poisson distribution accurately describes the data, this high-count signal could also be approximated as Gaussian, ${ }^{\mathbf{4 2 , 4 3}}$ similar to high-count signals for pulse-counting MS detectors. ${ }^{44}$

Since low-count TOFMS signals are best described with a compound Poisson distribution, the most accurate approach to establish a NP-detection threshold is to calculate net critical values specifically for this given distribution, i.e. $L_{\mathrm{C}(\mathrm{ADC})}$. Unlike Gaussian-distributed background noise, which has wellestablished $z$-scores that allow direct calculation of $L_{\mathrm{C}}$ based on measurement or estimation of $\sigma,,^{24,28}$ the compound Poisson distribution obtained with TOFMS detection does not follow a known distribution and is dependent on an empirical detector response curve, and thus cannot be simply calculated. For this reason, we estimate the relationship between $L_{\mathrm{C}(\mathrm{ADC})}$ and $\lambda_{\text {bkgd }}$ for a given false-positive rate $(\alpha)$ through Monte Carlo simulation of compound Poisson distributed TOF signals for a range of $\lambda_{\text {bkgd }}$ values (from $0.25-25$ counts per acq) and a measured SIS histogram. ${ }^{27}$ Importantly, because the compound Poisson distribution changes as a function of shape of the TOF detection system response curve (i.e. SIS histogram), routine measurements and calibration of the SIS are required to define a reliable $L_{\mathrm{C}(\mathrm{ADC})}$. For example, SIS histograms likely vary between instruments or could change due to detector aging.

For measurements reported here, $L_{\mathrm{C}(\mathrm{ADC})}$, is defined in eqn (1), where the $\alpha$ value is set conservatively to $0.0001(0.01 \%)$.

$$
L_{\mathrm{C}(\mathrm{ADC})}=4.34 \sqrt{\lambda_{\mathrm{bkgd}}}+2.27
$$

To use $L_{\mathrm{C}(\mathrm{ADC})}$ as a detection decision to identify NPs, it is more convenient to work with the gross signal critical value $\left(S_{\mathrm{C}(\mathrm{ADC})}\right)$, which is defined as the net critical value $L_{\mathrm{C}(\mathrm{ADC})}$ plus $\lambda_{\text {bkgd }}$ (see eqn (2)). To determine the analyte-mass detection decision $\left(X_{\mathrm{C}(\mathrm{ADC})}\right)$, the net signal critical value $\left(L_{\mathrm{C}(\mathrm{ADC})}\right)$ is divided by the net absolute mass sensitivity $\left(A_{\mathrm{i}, \mathrm{drop}}\right)$ obtained from microdroplet standards, as seen in eqn (3).

$$
\begin{gathered}
S_{\mathrm{C}(\mathrm{ADC})}=\lambda_{\mathrm{bkgd}}+L_{\mathrm{C}(\mathrm{ADC})}=\lambda_{\mathrm{bkgd}}+4.34 \sqrt{\lambda_{\mathrm{bkgd}}}+2.27 \\
X_{\mathrm{C}(\mathrm{ADC})}=\frac{L_{\mathrm{C}(\mathrm{ADC})}}{A_{\mathrm{i}, \mathrm{drop}}}=\frac{4.34 \sqrt{\lambda_{\mathrm{bkgd}}}+2.27}{A_{\mathrm{i}, \mathrm{drop}}}
\end{gathered}
$$

For determination of $L_{\mathrm{C}(\mathrm{ADC})}$ and $S_{\mathrm{C}(\mathrm{ADC})}$, we set $\alpha=0.01 \%$ because our analyses of $\mathrm{Au}-\mathrm{Ag}$ and $\mathrm{Au}$ NPs have relatively few NP events compared to the background. For each sp-ICPTOFMS analysis, we measure about 90000 data points, so $\alpha=$ $0.01 \%$ predicts nine false-NP signal events. Considering an acceptable false-detection rate of $10 \%$ of the total NPs, our chosen $\alpha$ should enable quantitative counting of NPs of down to $\sim 90$ measured NP events. To detect lower numbers of NPs quantitatively, $\alpha$ would need to be lowered, which would elevate $S_{\mathrm{C}(\mathrm{ADC})}$ and might cause non-detection of analyte NPs. In general, the choice of $\alpha$ for NP detection is somewhat arbitrary, and the analyst must find a useful compromise between selection of acceptable false-positive rates and NP-detection threshold.

\subsection{Case study: measuring Au-Ag NPs signal distributions}

To investigate the accuracy of $S_{\mathrm{C}(\mathrm{ADC})}$ for NP identification, we measured $60 \mathrm{~nm}$ diameter $\mathrm{Au}-\mathrm{Ag}$ core-shell NPs by sp-ICPTOFMS and then used $S_{\mathrm{C}(\mathrm{ADC})}$ as a NP-detection threshold to identify the Au-NP signals. Because the $\mathrm{Ag}$ shell contains roughly four times the mass of the Au core, the $\mathrm{Ag}$ signal from each NP is well above the background, and can be used as a control to record the true number of $\mathrm{Au}-\mathrm{Ag}$ NPs introduced into the plasma. With this Ag control, we are able to assess the accuracy of $S_{\mathrm{C}(\mathrm{ADC})}$ as a NP-detection criterion for the lowercount Au-NP signals, both in terms of recovery of $\mathrm{Au}-\mathrm{Ag} \mathrm{NP}$ counts and false Au-NP signals.

In Fig. 2, we plot a portion of the $\mathrm{Au}-\mathrm{Ag} \mathrm{NP}$ time trace, as well as the signal-intensity frequency histograms for ${ }^{107+109} \mathrm{Ag}$ and ${ }^{197} \mathrm{Au}$, respectively (see Fig. $2 \mathrm{~b}$ and c). As displayed in Fig. 2b, the Ag NP signal distribution is baseline separated from the dissolved background signals, and a visually set threshold value of 10 counts was used to distinguish NP signals from the background. However, for ${ }^{197} \mathrm{Au}^{+}$signals, there is no clear separation between the dissolved background and NP distributions. Instead, we find a bimodal, but uninterrupted, distribution that has a very similar shape to the distribution observed in Fig. 1a. Again, most ion signals occupy the zero-count bin and a right-screwed distribution is present at slightly higher count values. To determine the fraction of the Au signal associated with the dissolved background, we fit the Au-signal distribution with our Monte Carlo method to find $\lambda_{\text {bkgd }}$ and $N_{\text {acq }}$. Then, we used this $\lambda_{\text {bkgd }}$ value to calculate $S_{\mathrm{C}(\mathrm{ADC})}$. As shown in Fig. 3, thresholding Au-signals based on $S_{\mathrm{C}(\mathrm{ADC})}=2.77$ counts provides accurate identification of Au-NP signals $(98.3 \%$ NP recovery). The detection decision level in terms of Au-mass $\left(X_{\mathrm{C}(\mathrm{ADC})}\right)$ is $68 \mathrm{ag}$, or $\sim 19 \mathrm{~nm}$ in diameter. We do not measure $100 \%$ of the Au NPs because some of the Au-Au NPs produce ${ }^{197} \mathrm{Au}^{+}$signals of less than 2.77 counts. Based on concomitance of found Au-NP signals and Ag-NP signals, we determined that 10 of the identified ${ }^{197} \mathrm{Au}^{+}$signals found above $S_{\mathrm{C}(\mathrm{ADC})}$ were false positives, which is slightly less than the 26 predicted based on our assignment of $\alpha=0.01 \%$.

For comparison, we also attempted to identify Au-NP signals based on conventional iterative outlier analysis with outlier thresholds of $\mu+3 \sigma$ and $\mu+5 \sigma$. In this iterative outlier analysis, we found that data with zero counts had to be removed from the dataset to prevent the threshold-value from converging to zero. However, in our particular case, when zero-count data was removed, the background : NP number ratio was low $(\sim 2: 1)$ and the iterative outlier analysis algorithm failed to accurately distinguish background and NP distributions, which resulted in artificially high NP-detection thresholds and low Au-NP recoveries (see Fig. 3a).

On the other hand, we also determined $\mu+3 \sigma$ and $\mu+5 \sigma$ NPdetection thresholds based on fitting the non-zero background histogram with a Gaussian function. This Gaussian-fitting procedure produced more realistic results than the iterative outlier analysis, with NP recoveries of $103 \%$ and $99 \%$ for $\mu+3 \sigma$ and $\mu+5 \sigma$ thresholds, respectively. However, it should be noted that if the non-zero-background was truly Gaussian distributed, 
a)

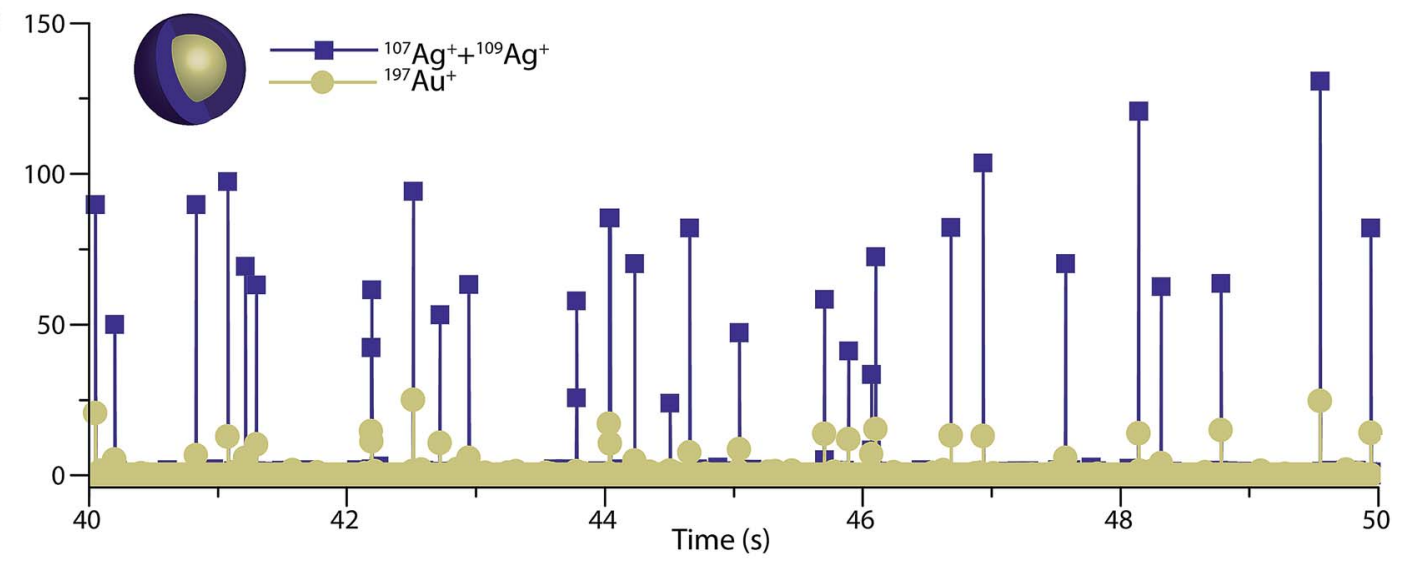

b)

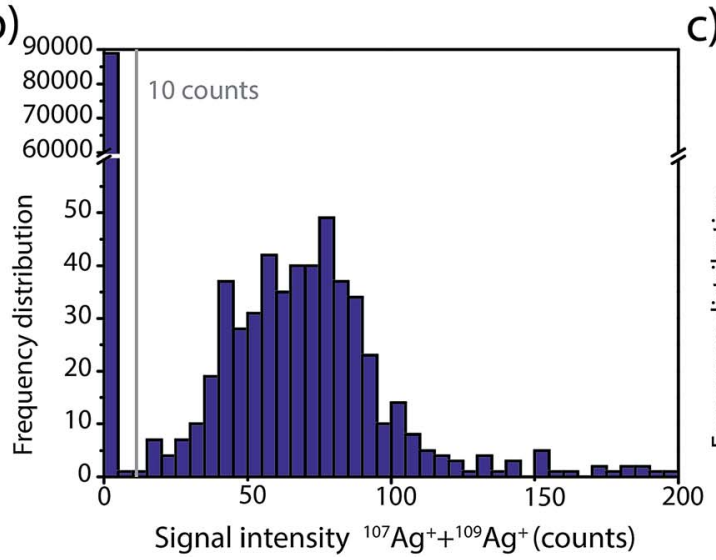

c)

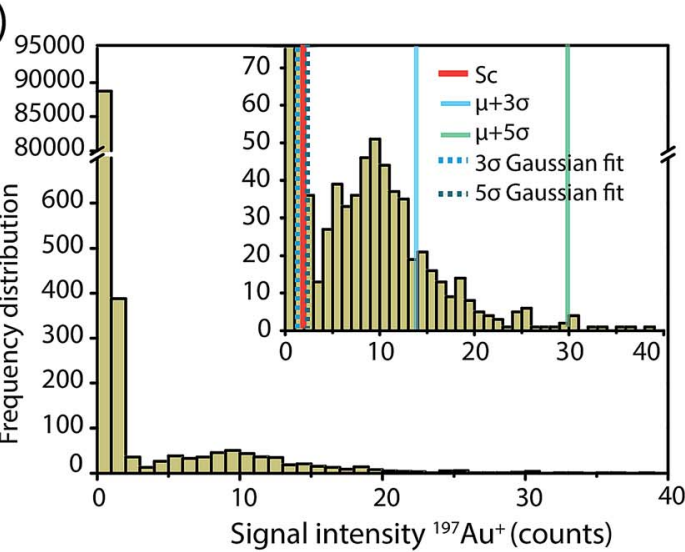

Fig. 2 (a) sp-ICP-TOFMS time trace of the Au-Ag NPs. (b) and (c) Signal-intensity frequency distributions of the measured Au-Ag NPs for the combined signal of isotopes ${ }^{107} \mathrm{Ag}$ and ${ }^{109} \mathrm{Ag}$ from the shell (b) and for the $30 \mathrm{~nm}$ Au core (c). As the Ag signals are easily distinguished from the background, a NP detection threshold was set at 10 counts. However, for Au signals different decision threshold approaches were compared. The accuracy of the Au NPs identification can be checked by concomitance with Ag signals.

a $\mu+3 \sigma$ detection threshold should produce $0.135 \%$ false positives. Consequently, as our non-zero-background is composed of $\sim 3000$ data points, one would predict only 4 false- positive NP signals. Instead, the $\mu+3 \sigma$ detection decision produces 38 false-positive Au-NP signals. Deletion of zero-count data - which is the major signal level in the background-prior

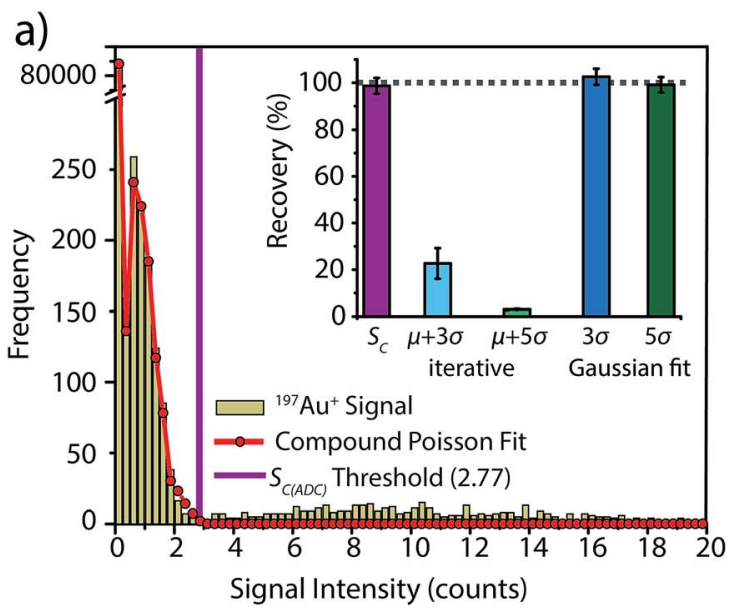

b)

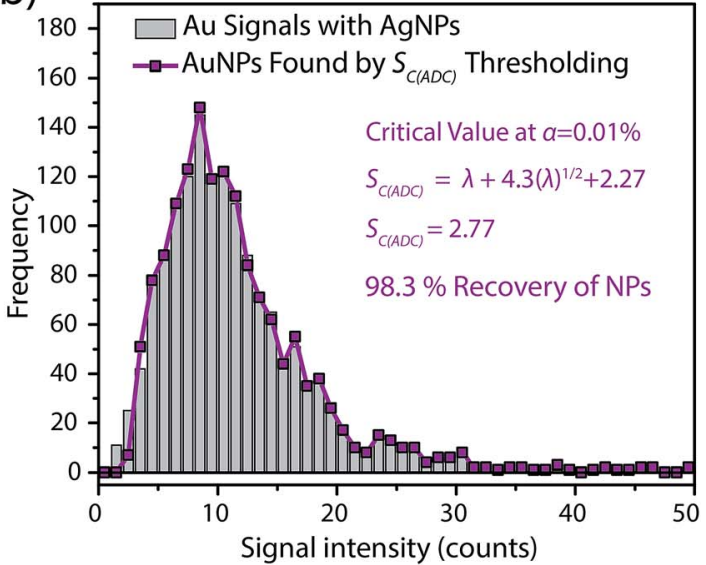

Fig. 3 (a) Histogram of the ${ }^{197} \mathrm{Au}^{+}$signals with an overlay of the simulated background-signal distribution from our Monte Carlo approach and $\lambda_{\text {bkgd }}=0.012 \mathrm{cts}$ per acq; the $S_{C(A D C)}$ NP-detection threshold is also provided. An overview of the recoveries obtained using the different NPdetection threshold approaches is presented in the inset. Error bars represent the standard deviation of three replicates. (b) Histogram of the ${ }^{197} \mathrm{Au}^{+}$signals identified above $S_{C(A D C)}$. True Au-NP signals are determined by ${ }^{197} \mathrm{Au}^{+}$concomitant with the control ${ }^{107+109} \mathrm{Ag}^{+}$signals. 
a)

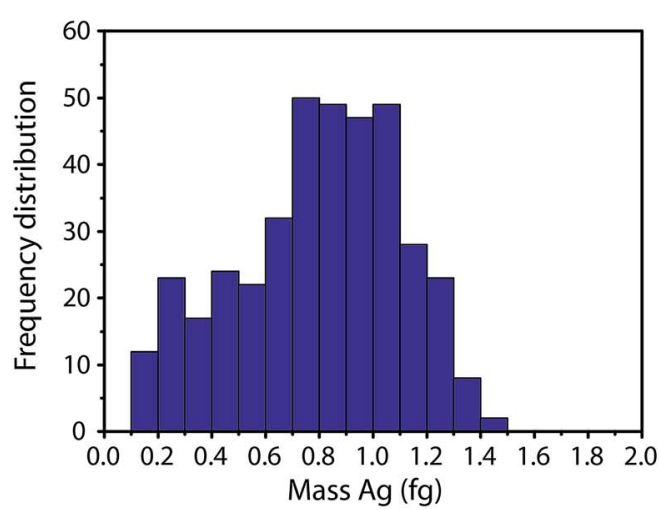

b)

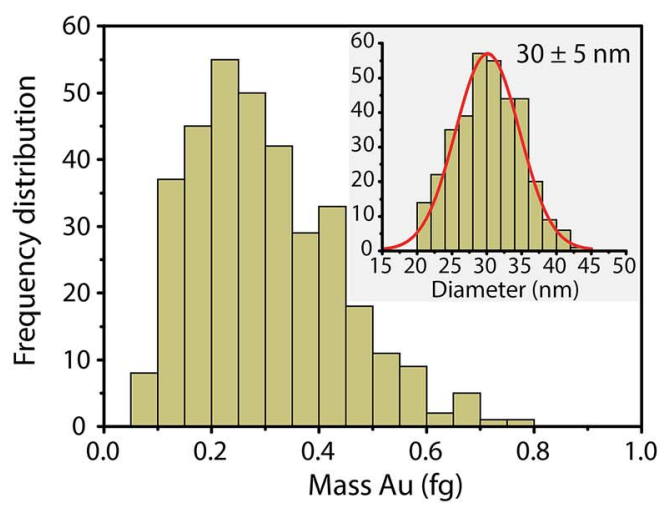

Fig. 4 Calibrated mass frequency distributions of the Ag shell (a) and Au core (b). The Au mass frequency distribution was further converted into a size frequency distribution assuming a spherical shape and the same density as the bulk, and fitted with a normal distribution yielding a mean size of $30 \pm 5 \mathrm{~nm}$

to background variance calculation alters the true shape of the background distribution. This signal truncation causes the calculated detection decisions to be based on fitting of incomplete datasets, and it is difficult to predict how characteristics such as background : NP event ratios or instrument response function could affect the performance of these detection decisions. The use of $S_{\mathrm{C}(\mathrm{ADC})}$ instead of $\sigma$-based NP-detection criteria takes into account the compound Poisson nature of the ICPTOFMS data to provide a more complete description of the dissolved background signal, and therefore offers more robust NP detection with predictable false positive rates.

In Fig. 4, we report the quantified mass of $\mathrm{Ag}$ and $\mathrm{Au}$ in each $\mathrm{Au}-\mathrm{Ag}$ core-shell NP based on online microdroplet calibration, which was used to establish absolute sensitivities for ${ }^{107+109} \mathrm{Ag}$ and ${ }^{197} \mathrm{Au}^{32,33}$ Prior to quantification, Au-NP signals were identified based on the $S_{\mathrm{C}(\mathrm{ADC})}$ threshold. As seen, the determined element mass for both the $\mathrm{Ag}$ shell and the $\mathrm{Au}$ core matches expectations (see Table 1). For the Au core, we also plot the diameter frequency distribution-which again matches expectations. An important point to highlight here is that the use of a NP-detection threshold (i.e. $S_{\mathrm{C}(\mathrm{ADC})}$ ) enables the identification of NPs on a particle-to-particle basis. Hence, for each $\mathrm{Au}$ NP detected above $S_{\mathrm{C}(\mathrm{ADC})}$, the corresponding mass of Ag in that NP can be determined, which is an important requirement for studies that classify NPs based on their multi-elemental composition. ${ }^{35,36,38}$

\subsection{Measuring single-particle distribution: application to nominal $20 \mathrm{~nm}$ Au NPs}

In the previous section, we demonstrated that $S_{\mathrm{C}(\mathrm{ADC})}$ is an accurate NP-detection criterion and allows for excellent NP recovery in cases where minimal overlap occurs between the dissolved background and NP signal distributions. Now, we will move on to the measurement of $20 \mathrm{~nm}$ Au NPs, which, based on sensitivity measured with microdroplet standards, are expected a)

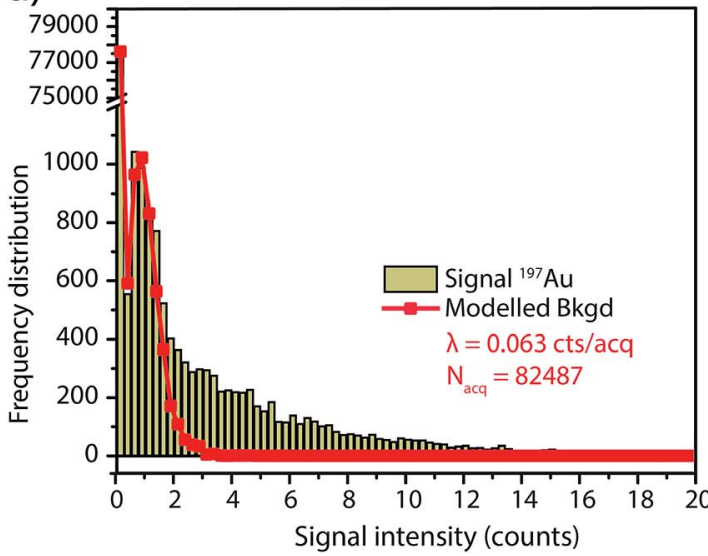

b)

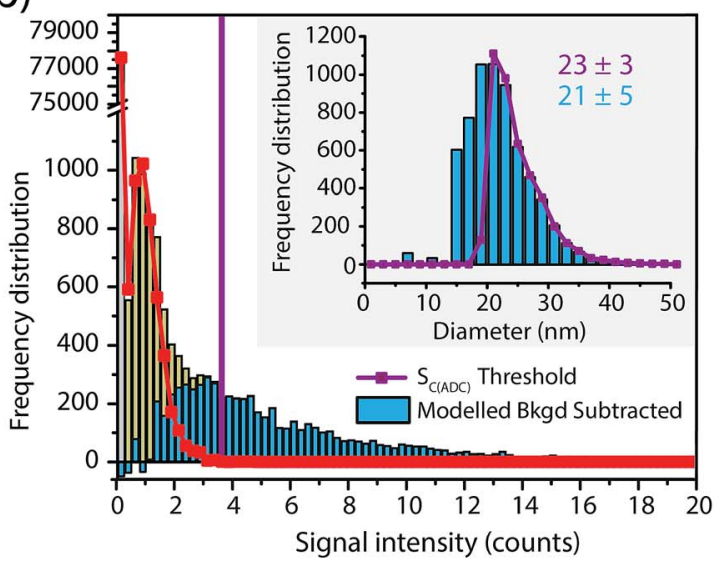

Fig. 5 (a) Signal frequency distribution of ${ }^{197} \mathrm{Au}^{+}$signals; there is no clear separation of the signals from the $20 \mathrm{~nm}$ Au NPs and the dissolved background fraction. The ${ }^{197} \mathrm{Au}^{+}$signal distribution is fitted by Monte Carlo simulation to predict the dissolved background distribution. As seen, the simulated background distribution does not account for all ${ }^{197} \mathrm{Au}^{+}$signals; the remaining signals can be attributed to NPs. (b) NPs are identified using two approaches: $S_{C(A D C)}$ as the detection threshold and by subtraction the modelled background. The corresponding size distributions using both NP-detection approaches yield a mean sizes of $23 \pm 3 \mathrm{~nm}$ and $21 \pm 5 \mathrm{~nm}$, respectively. 

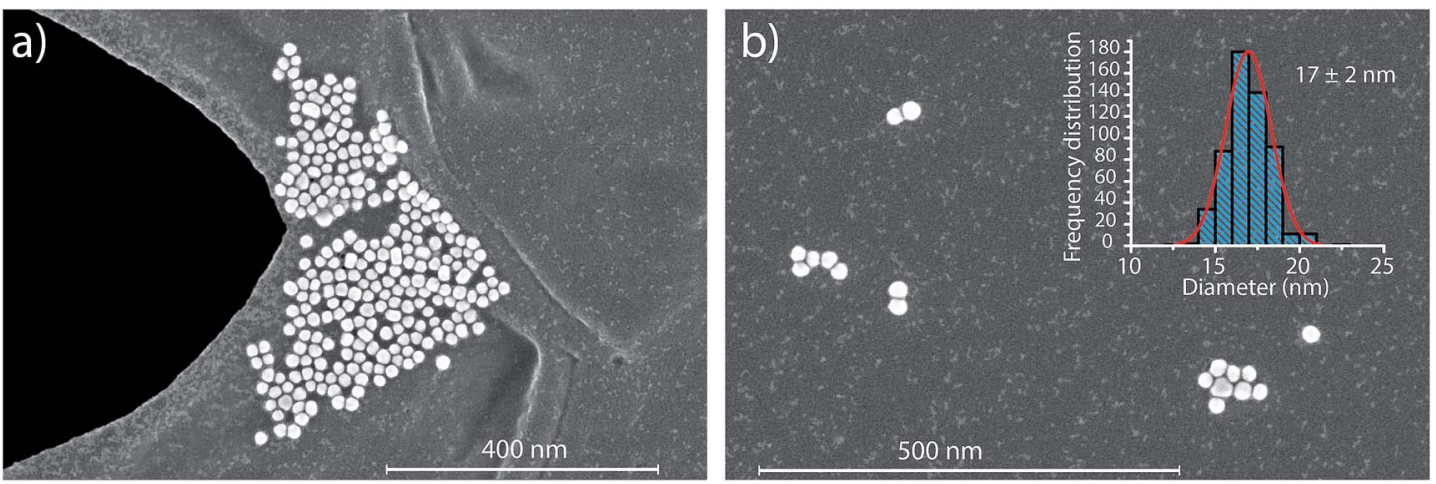

Fig. 6 (a) S(T)EM images of the nominal $20 \mathrm{~nm}$ Au NPs under investigation. (b) After image analysis, 562 NPs were binned into in a size histogram and fitted with a normal distribution, yielding a mean size of $17 \pm 2 \mathrm{~nm}$.

to produce $\sim 3.8$ counts per NP. As displayed in Fig. 5 , the lowcount signals measured from the $20 \mathrm{~nm}$ Au NPs are recognizable only as a slight shoulder on the Au-signal histogram. Here again, we fit the signal distribution via our Monte Carlo approach to determine the most likely $\lambda_{\text {bkgd }}$ and $N_{\text {acq }}$ that describe the dissolved background distribution (see Fig. 5a). However, for this Au-signal distribution, there is no clear separation of the dissolved background and the NP distributions, so thresholding data based on $S_{\mathrm{C}(\mathrm{ADC})}$ will result in the loss of many NP-signals that are clearly not accounted for by the compound Poisson fit. To overcome this limitation, we subtracted the Monte Carlo simulated background from the measured signal distribution. As seen in Fig. 5b, this subtraction enables recovery of NP signals with intensities lower than $S_{\mathrm{C}(\mathrm{ADC})}$. Unfortunately, because these Au NPs are not coated with Ag as before, we cannot directly assess percent of NP recovery or false positives. Nonetheless, after calibrating ${ }^{197} \mathrm{Au}^{+}$signals to NP diameters, it becomes apparent that signals uncovered through modelled background subtraction do match well with the expected size distribution of the $20 \mathrm{~nm} \mathrm{Au} \mathrm{NPs} \mathrm{(cf.} \mathrm{Fig.} \mathrm{6).} \mathrm{While} \mathrm{modelled}$ background subtraction seems to allow extraction of NP signals that overlap with the dissolved background distribution, contrary to $S_{\mathrm{C}(\mathrm{ADC})}$ NP-detection thresholding, it cannot be used to analyze particles on a particle-to-particle basis. Indeed, after modelled background subtraction, information on individual NPs is lost and only distribution-based information remains, such as size distribution or mass distribution.

Sizing results are compared with S(T)EM measurements presented in Fig. 6. From the S(T)EM measurements, it can be observed that not all NPs are as spherical as initially expected. Large non-spherical particles were not accounted for in the $\mathrm{S}(\mathrm{T})$ EM data evaluation, which could explain the slightly lower average $\mathrm{Au}$ NP diameter found by S(T)EM image analysis compare to sp-ICP-TOFMS results.

\section{Conclusion}

In a previous publication, we investigated the nature of lowcount TOFMS signals using microdroplets as proxy for NPs; ${ }^{27}$ here, we've applied the concept of compound Poisson background modelling through Monte Carlo simulation for the analysis of Au-Ag core-shell NPs and $20 \mathrm{~nm}$ Au NPs by sp-ICPTOFMS. We demonstrated that, with sp-ICP-TOFMS, individual NPs are most accurately detected by using the critical value $S_{\mathrm{C}(\mathrm{ADC})}$ as a detection threshold. Through the measurement of $\mathrm{Au}-\mathrm{Ag}$ core-shell NPs, we showed that the decision threshold $S_{\mathrm{C}(\mathrm{ADC})}$ yields a $98.3 \%$ NPs recovery. In contrast, the commonly applied iterative outlier analysis approach for NP detection yielded only $23 \%$ and $2 \%$ recovery, using $\mu+3 \sigma$ and $\mu+5 \sigma$, respectively. In our example, iterative outlier analysis fails because it assumes that the background is normally distributed and that there are many more background data than NP data. There are certainly cases where iterative outlier analysis would produce reasonable NP identification with sp-ICP-TOFMS; however, the underlying assumption that background data is normally distributed is false, and this can lead to spurious results.

In addition to developing NP-detection thresholds for spICP-TOFMS, we also demonstrate that, in cases where NP and the dissolved fractions overlap-such as in the case of $20 \mathrm{~nm}$ diameter Au NPs-the subtraction of a Monte Carlo modelled distribution from the measured signal distribution can be used to predict the fraction of NPs present below $S_{\mathrm{C}(\mathrm{ADC})}$. This treatment relies on very accurate Monte Carlo modelling of the background distribution and necessarily eliminates the information on individual particles. While modelled background subtraction is a promising approach, more work on characterizing uncertainty in Monte Carlo modelled distributions is required, especially if the approach is applied to sp-ICP-TOFMS measurement with low NP numbers.

In the presented method, the advantages of microdroplet calibration and the dual sample introduction system are combined with the new insights in TOFMS noise. Online calibration with microdroplets allows for wide flexibility in terms of materials and sizes of NPs, and also automatically compensates for plasma-related matrix effects. ${ }^{33}$ The development of more accurate noise characterization for ICP-TOFMS as demonstrated here, combined with dual sample introduction and online microdroplet calibration, make sp-ICP-TOFMS a promising method for the simultaneous quantification of diverse metal-containing NPs in terms of NP composition and number concentration in complex sample matrices. 


\section{Conflicts of interest}

There are no conflicts of interest to declare.

\section{Acknowledgements}

The authors thank Roland Mäder from the ETH mechanical workshop for manufacturing custom pieces necessary for the microdroplet introduction system and the dual inlet setup. L. H. acknowledges funding from Swiss National Science Foundation (SNSF) Project no. 200021 162870/1. A. G.-G. acknowledges funding through an Ambizione grant of the SNSF, project no. PZ00P2_174061. The authors acknowledge support of the Scientific Center for Optical and Electron Microscopy (ScopeM) of the Swiss Federal Institute of Technology ETHZ.

\section{References}

1 H. Wei, O. T. Bruns, M. G. Kaul, E. C. Hansen, M. Barch, A. Wiśniowska, O. Chen, Y. Chen, N. Li and S. Okada, Exceedingly small iron oxide nanoparticles as positive MRI contrast agents, Proc. Natl. Acad. Sci. U. S. A., 2017, 114, 2325-2330.

2 E. H. Chang, J. B. Harford, M. A. Eaton, P. M. Boisseau, A. Dube, R. Hayeshi, H. Swai and D. S. Lee, Nanomedicine: past, present and future-a global perspective, Biochem. Biophys. Res. Commun., 2015, 468, 511-517.

3 L. Zhang, F. Gu, J. Chan, A. Wang, R. Langer and O. Farokhzad, Nanoparticles in medicine: therapeutic applications and developments, Clin. Pharmacol. Ther., 2008, 83, 761-769.

4 R. D. Handy, G. Cornelis, T. Fernandes, O. Tsyusko, A. Decho, T. Sabo-Attwood, C. Metcalfe, J. A. Steevens, S. J. Klaine and A. A. Koelmans, Ecotoxicity test methods for engineered nanomaterials: practical experiences and recommendations from the bench, Environ. Toxicol. Chem., 2012, 31, 15-31.

5 O. J. Osborne, S. Lin, C. H. Chang, Z. Ji, X. Yu, X. Wang, S. Lin, T. Xia and A. E. Nel, Organ-specific and sizedependent $\mathrm{Ag}$ nanoparticle toxicity in gills and intestines of adult zebrafish, ACS Nano, 2015, 9, 9573-9584.

6 C. Degueldre and P. Y. Favarger, Colloid analysis by single particle inductively coupled plasma-mass spectroscopy: a feasibility study, Colloids Surf., A, 2003, 217, 137-142.

7 F. Laborda, E. Bolea and J. Jiménez-Lamana, Single Particle Inductively Coupled Plasma Mass Spectrometry: A Powerful Tool for Nanoanalysis, Anal. Chem., 2014, 86, 2270-2278.

8 H. E. Pace, N. J. Rogers, C. Jarolimek, V. A. Coleman, C. P. Higgins and J. F. Ranville, Determining Transport Efficiency for the Purpose of Counting and Sizing Nanoparticles via Single Particle Inductively Coupled Plasma Mass Spectrometry, Anal. Chem., 2011, 83, 93619369.

9 M. Hadioui, C. Peyrot and K. J. Wilkinson, Improvements to Single Particle ICPMS by the Online Coupling of Ion Exchange Resins, Anal. Chem., 2014, 86, 4668-4674.
10 L. Fréchette-Viens, M. Hadioui and K. J. Wilkinson, Quantification of $\mathrm{ZnO}$ nanoparticles and other $\mathrm{Zn}$ containing colloids in natural waters using a high sensitivity single particle ICP-MS, Talanta, 2019, 200, 156162.

$11 \mathrm{H}$. El Hadri and V. A. Hackley, Investigation of cloud point extraction for the analysis of metallic nanoparticles in a soil matrix, Environ. Sci.: Nano, 2017, 4, 105-116.

12 S. T. Kim, H. K. Kim, S. H. Han, E. C. Jung and S. Lee, Determination of size distribution of colloidal $\mathrm{TiO}_{2}$ nanoparticles using sedimentation field-flow fractionation combined with single particle mode of inductively coupled plasma-mass spectrometry, Microchem. J., 2013, 110, 636642.

13 K. Proulx and K. J. Wilkinson, Separation, detection and characterisation of engineered nanoparticles in natural waters using hydrodynamic chromatography and multimethod detection (light scattering, analytical ultracentrifugation and single particle ICP-MS), Environ. Chem., 2014, 11, 392-401.

14 B. Franze, I. Strenge and C. Engelhard, Separation and detection of gold nanoparticles with capillary electrophoresis and ICP-MS in single particle mode (CE-SPICP-MS), J. Anal. At. Spectrom., 2017, 32, 1481-1489.

$15 \mathrm{~J}$. Tuoriniemi, G. Cornelis and M. Hassellov, A new peak recognition algorithm for detection of ultra-small nanoparticles by single particle ICP-MS using rapid time resolved data acquisition on a sector-field mass spectrometer, J. Anal. At. Spectrom., 2015, 30, 1723-1729.

16 P. N. Shaw and A. Donard, Nano-particle analysis using dwell times between $10 \mu \mathrm{s}$ and $70 \mu \mathrm{s}$ with an upper counting limit of greater than $3 \times 107$ cps and a gold nanoparticle detection limit of less than $10 \mathrm{~nm}$ diameter, J. Anal. At. Spectrom., 2016, 31, 1234-1242.

17 M. S. Jiménez, M. T. Gómez, E. Bolea, F. Laborda and J. Castillo, An approach to the natural and engineered nanoparticles analysis in the environment by inductively coupled plasma mass spectrometry, Int. J. Mass Spectrom., 2011, 307, 99-104.

18 D. M. Mitrano, A. Barber, A. Bednar, P. Westerhoff, C. P. Higgins and J. F. Ranville, Silver nanoparticle characterization using single particle ICP-MS (SP-ICP-MS) and asymmetrical flow field flow fractionation ICP-MS (AF4-ICP-MS), J. Anal. At. Spectrom., 2012, 27, 1131-1142.

19 F. Laborda, J. Jimenez-Lamana, E. Bolea and J. R. Castillo, Critical considerations for the determination of nanoparticle number concentrations, size and number size distributions by single particle ICP-MS, J. Anal. At. Spectrom., 2013, 28, 1220-1232.

$20 \mathrm{~J}$. Tuoriniemi, G. Cornelis and M. Hassellöv, Size Discrimination and Detection Capabilities of SingleParticle ICPMS for Environmental Analysis of Silver Nanoparticles, Anal. Chem., 2012, 84, 3965-3972.

$21 \mathrm{G}$. Cornelis and M. Hassellov, A signal deconvolution method to discriminate smaller nanoparticles in single particle ICP-MS, J. Anal. At. Spectrom., 2014, 29, 134-144. 
22 X. Bi, S. Lee, J. F. Ranville, P. Sattigeri, A. Spanias, P. Herckes and P. Westerhoff, Quantitative resolution of nanoparticle sizes using single particle inductively coupled plasma mass spectrometry with the $K$-means clustering algorithm, $J$. Anal. At. Spectrom., 2014, 29, 1630-1639.

23 D. Mozhayeva and C. Engelhard, A quantitative nanoparticle extraction method for microsecond time resolved singleparticle ICP-MS data in the presence of a high background, J. Anal. At. Spectrom., 2019, DOI: 10.1039/c9ja00042a.

24 E. Voigtman, Limits of Detection in Chemical Analysis, John Wiley \& Sons, Inc., USA, 2017.

25 C. Degueldre, P. Y. Favarger and S. Wold, Gold colloid analysis by inductively coupled plasma-mass spectrometry in a single particle mode, Anal. Chim. Acta, 2006, 555, 263268.

26 J. Navratilova, A. Praetorius, A. Gondikas, W. Fabienke, F. von der Kammer and T. Hofmann, Detection of Engineered Copper Nanoparticles in Soil Using Single Particle ICP-MS, Int. J. Environ. Res. Public Health, 2015, 12, 15020.

27 A. Gundlach-Graham, L. Hendriks, K. Mehrabi and D. Günther, Monte Carlo Simulation of Low-Count Signals in Time-of-Flight Mass Spectrometry and its Application to Single-Particle Detection, Anal. Chem., 2018, 90, 1184711855.

28 L. A. Currie, Limits for qualitative detection and quantitative determination. Application to radiochemistry, Anal. Chem., 1968, 40, 586-593.

29 L. A. Currie, in Radioactivity in the Environment, ed. P. P. Pavel, Elsevier, 2008, vol. 11, pp. 49-135.

30 L. A. Currie, Nomenclature in evaluation of analytical methods including detection and quantification capabilities, Pure Appl. Chem., 1995, 67, 1699-1723.

31 B. Ramkorun-Schmidt, S. A. Pergantis, D. EstebanFernández, N. Jakubowski and D. Günther, Investigation of a Combined Microdroplet Generator and Pneumatic Nebulization System for Quantitative Determination of Metal-Containing Nanoparticles Using ICPMS, Anal. Chem., 2015, 87, 8687-8694.

32 L. Hendriks, A. Gundlach-Graham and D. Günther, Analysis of Inorganic Nanoparticles by Single-Particle Inductively Coupled Plasma Time-of-Flight Mass Spectrometry, CHIMIA International Journal for Chemistry, 2018, 72, 221226.

33 L. Hendriks, B. Ramkorun-Schmidt, A. Gundlach-Graham, J. Koch, R. N. Grass, N. Jakubowski and D. Günther, Single-particle ICP-MS with online microdroplet calibration: toward matrix independent nanoparticle sizing, J. Anal. At. Spectrom., 2019, 34, 716-728.

34 O. Borovinskaya, S. Gschwind, B. Hattendorf, M. Tanner and D. Günther, Simultaneous Mass Quantification of
Nanoparticles of Different Composition in a Mixture by Microdroplet Generator-ICPTOFMS, Anal. Chem., 2014, 86, 8142-8148.

35 A. Praetorius, A. Gundlach-Graham, E. Goldberg, W. Fabienke, J. Navratilova, A. Gondikas, R. Kaegi, D. Günther, T. Hofmann and F. von der Kammer, Singleparticle multi-element fingerprinting (spMEF) using inductively-coupled plasma time-of-flight mass spectrometry (ICP-TOFMS) to identify engineered nanoparticles against the elevated natural background in soils, Environ. Sci.: Nano, 2017, 4, 307-314.

36 A. Gondikas, F. von der Kammer, R. Kaegi, O. Borovinskaya, E. Neubauer, J. Navratilova, A. Praetorius, G. Cornelis and T. Hofmann, Where is the nano? Analytical approaches for the detection and quantification of $\mathrm{TiO}_{2}$ engineered nanoparticles in surface waters, Environ. Sci.: Nano, 2018, 5, 313-326.

37 S. Naasz, S. Weigel, O. Borovinskaya, A. Serva, C. Cascio, A. K. Undas, F. C. Simeone, H. J. P. Marvin and R. J. B. Peters, Multi-element analysis of single nanoparticles by ICP-MS using quadrupole and time-offlight technologies, J. Anal. At. Spectrom., 2018, 33, 835-845.

38 F. Loosli, J. Wang, S. Rothenberg, M. Bizimis, C. Winkler, O. Borovinskaya, L. Flamigni and M. Baalousha, Sewage spills are a major source of titanium dioxide engineered (nano)-particle release into the environment, Environ. Sci.: Nano, 2019, 6, 763-777.

39 J. Koch, L. Flamigni, S. Gschwind, S. Allner, H. Longerich and D. Gunther, Accelerated evaporation of microdroplets at ambient conditions for the on-line analysis of nanoparticles by inductively-coupled plasma mass spectrometry, J. Anal. At. Spectrom., 2013, 28, 1707-1717.

40 L. Flamigni, Doctor of Sciences, Doctoral Thesis, ETH Zürich, 2014.

41 S. Gschwind, L. Flamigni, J. Koch, O. Borovinskaya, S. Groh, K. Niemax and D. Gunther, Capabilities of inductively coupled plasma mass spectrometry for the detection of nanoparticles carried by monodisperse microdroplets, $J$. Anal. At. Spectrom., 2011, 26, 1166-1174.

42 K. L. Hunter and R. W. Stresau, Influence of Detector Pulse Height Distribution on Abundance Accuracy in TOFMS, ETP Electron Multipliers, Australia, 1998.

43 A. Ipsen, Derivation from First Principles of the Statistical Distribution of the Mass Peak Intensities of MS Data, Anal. Chem., 2015, 87, 1726-1734.

44 F. Laborda, J. Medrano and J. R. Castillo, Quality of quantitative and semiquantitative results in inductively coupled plasma mass spectrometry, J. Anal. At. Spectrom., 2001, 16, 732-738. 